\title{
Extracorporeal Cardiopulmonary Resuscitation in a Cardiac Arrest Patient Due to Digoxin Overdose Who Was Awake During Conventional Cardiopulmonary Resuscitation
}

Yunus Emre ÖZLÜER*, Mücahit AVCiL*, Duygu EGE*, Çağaç YETiş*, Adnan Menderes University Hospital Department of Emergency Medicine, Aydın/TURKEY

doi.org/10.33706/jemcr.658218

\begin{abstract}
Introduction: Digoxin overdose, which may cause rhythm disturbances, such as atrioventricular blocks, can ultimately cause cardiac arrest. Extracorporeal life support may provide time until specific antidotes show an effect.

Case Report: A 75-year-old male patient presented with dyspnea with a known history of congestive heart failure. His medications included digoxin, and his initial electrocardiography showed an idioventricular rhythm. His digoxin level was over the upper normal limit, and he had hyperkalemia $(7.9 \mathrm{Meq} / \mathrm{L})$. The patient collapsed during conventional therapies, including insulinglucose infusion, atropine administration, and catheterization for emergency hemodialysis, and while awaiting digoxin-specific fragment antibodies. During mechanical cardiopulmonary resuscitation, the patient was awake and had a Full Outline of Unresponsiveness score of 8, which led us to initiate veno-arterial extracorporeal membrane oxygenation.

Conclusion: Extracorporeal Cardiopulmonary Resuscitation, might be a useful option for intoxicated patients when conventional therapies fail. However, more experience is needed for the increase in survival rate.
\end{abstract}

Keywords: Extracorporeal Cardiopulmonary Resuscitation,Digoxin,Overdose,ECMO

\section{Introduction}

Digoxin is a cardiac glycoside that causes a decreased heart rate, increased myocardial contraction, and deceleration in Atrioventricular (AV) nodal conduction (1). Thus, digoxin is widely used for treating congestive heart failure (CHF) with low ejection fraction and accelerated ventricular response (2). However, digoxin has a narrow therapeutic index, and toxicity may occur during chronic use, especially in elderly patients with decreased renal excretion of digoxin (3). Signs of toxicity, including nausea, vomiting, anorexia, scotomas, AV conduction disorders, and even asystole, occur more frequently when the serum digoxin concentration is above $2 \mathrm{ng} / \mathrm{mL}$, but they also may be present when the concentration within normal limits (4). Digoxin-specific fragment antibodies (DigiFab) are an antidotal therapy that is used in association with conventional adjunct measures. Extracorporeal life support with veno-arterial extracorporeal membrane oxygenation (VA ECMO) may provide extra time for antidotal and supportive interventions to take effect.

With this case report we share our experience of extracorporeal cardiopulmonary resuscitation (E-CPR) in addition to 
conventional CPR procedures in a patient with cardiac arrest due to digoxin intoxication.

\section{Case Report}

A 75-year-old male with a history of chronic obstructive pulmonary disease (COPD), chronic renal failure (CRF), CHF, and coronary artery by-pass graft presented to the Emergency Department with dyspnea. His medications included clopidogrel, spironolactone, amlodipine, furosemide, atorvastatin, and digoxin. His vitals were as follows: blood pressure $138 / 42 \mathrm{mmHg}$, pulse rate $55 \mathrm{bpm}$, respiratory rate $12 / \mathrm{min}$, oxygen saturation $96 \%$, and body temperature $36^{\circ} \mathrm{C}$. An initial ECG revealed an idioventricular rhythm, with a rate of $40 \mathrm{bpm}$ (Figure 1). An arterial blood gas analysis showed metabolic acidosis with hyperkalemia $(7.9 \mathrm{mEq} / \mathrm{L})$. His laboratory findings were as follows: urea 227 $\mathrm{mg} / \mathrm{dl}$, creatinine $2.2 \mathrm{mg} / \mathrm{dl}$, and sodium 129 $\mathrm{mmol} / \mathrm{l}$. These findings were consistent with the patient's previous results. In addition, his serum digoxin level was $2.88 \mathrm{ng} / \mathrm{ml}$; therefore, the patient was diagnosed with digoxin intoxication. Prompt therapies for hyperkalemia and hemodynamic instability with intravenous calcium gluconate, insulinglucose and atropine were initiated. During the central venous catheterization of the left femoral vein for emergency hemodialysis, the patient developed cardiac arrest. The return of spontaneous circulation with the patient's initial rhythm was achieved after 10 minutes of cardiopulmonary resuscitation (CPR), which followed endotracheal intubation without any drug assistance. He was then transferred to our Critical Care Unit. DigiFab was not immediately available at our institution; thus, an intravenous lipid emulsion therapy was initiated at a bolus dose of 1.5 $\mathrm{mL} / \mathrm{kg}$ of lean body mass over 1 minute, followed by continuous infusion with a flow rate of $0.25 \mathrm{~mL} / \mathrm{kg} / \mathrm{min}$. Although we also placed a temporary transvenous pacemaker, even with the appropriate adjustments, the pacing was ineffective, and the patient had his second cardiac arrest minutes after the beginning of the pacing. During cardiac compressions with a Lucas $2 \AA$ (Stryker Medical, Michigan, US), the patient was awake with a Full Outline of Unresponsiveness (FOUR) Score of 8 (E2M2B4R0) and positive pupil reflexes without any sedative/paralyzing agent. However, during the pulse check, the patient had asystole with a FOUR score of 0 .

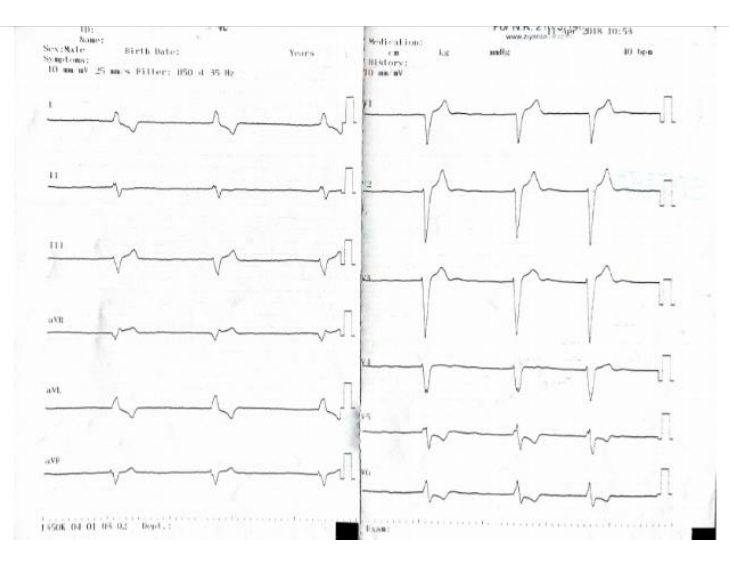

FIGURE 1. An initial ECG revealed an idioventricular rhythm, with a rate of $40 \mathrm{bpm}$

Thus, we decided to provide extracorporeal support and commenced VA ECMO during CPR. Cannulation was achieved with a left femoral cut-down, and continuous renal replacement therapy lines were integrated into the ECMO circuit. A flow rate of $3 \mathrm{~L} / \mathrm{min}$ was provided with an ECMO speed of 3,200 rpm along with noradrenaline, adrenaline, and dopamine infusion. At that point, the patient became unconscious, but positive pupil reflexes remained with a FOUR score of 4 (EOMOB4R0). On the 12th hour of extracorporeal support, the patient remained hypotensive and bradycardic, and all signs of life were lost. He had elevated lactate levels that were not consistent with life $(22 \mathrm{mmol} / \mathrm{L})$, despite the appropriate adjustment of the ECMO's pump rate. The decision for the termination of efforts was then made, and the patient was declared dead.

\section{Discussion}

Extracorporeal CPR (E-CPR) is an increasing trend in resuscitation medicine. However, there is still a lack of methodology, and the criteria for E-CPR candidates differ among institutions. For instance, Kagawa et al. stated that their institutional criteria for E-CPR include the following: patients between 18 and 74 years of age with an estimated duration of cardiac arrest-to-ECMO time of under 15 minutes and the inability to achieve 
a return of spontaneous circulation (ROSC) within 20 minutes (5). Our patient was 75 years old and had multiple comorbidities other than CHF, such as COPD and CRF. Furthermore, the ECMO device was not readily available at our institution at that moment, and it took 1.5 hours to be delivered from the nearest hospital. During this time, the patient was conventionally resuscitated using Lucas $2 \AA$. The main reason for initiating VA ECMO was that the patient was showing signs of life, and we were even able to communicate with him during CPR. Thus, we aimed to "buy some time" for the patient until the conventional therapies for hyperkalemia, bradycardia, and elimination of digoxin, such as insulin-glucose infusion, atropine, hemodialysis, and DigiFab, could show their effects.

In a recently published study, Wengenmayer et al. investigated the effect of the duration of mechanical CPR until the start of VA ECMO support (low-flow time) on survival between patients who survived and who did not (6). The authors found that the overall mean duration of low-flow time was $\sim 60$ minutes; in the survivor group, the mean low-flow time was 41.7 minutes, which was significant. Unfortunately, in our case, the arrest-toECMO time was $~ 90$ minutes, and we believe that this may have played a primary role in the survival of this patient. The main reason for this extended time was a lack of equipment and not having the ECMO device waiting on stand-by at our institution. In addition, even though ECMO providers on our staff were properly trained theoretically, this was our first E-CPR case. Real-life cases create a stressful environment that may lie beyond the scope of theoretical training alone. In this regard, more experience may be beneficial when treating patients who require extracorporeal life support.

The literature contains few cases of digitalis toxicity requiring $\mathrm{ECMO}$, but the toxicity in these cases was ingestion of yew containing Taxus baccata (7), foxglove (8), and pong-pong seeds (Cerbera odollam) (9). Hence, as far as we know, this is the first case of digitalis toxicity with chronic use of the drug itself in which extracorporeal life support was required, and the first E-CPR case reported by emergency physicians in Turkey. Digoxin is mainly excreted into urine by the kidneys. In elderly patients, for whom the prevalence of CRF is frequently high, dose adjustments are needed (3). In this context, we believe that the primary underlying mechanism of the toxicity was CRF, which decreased the elimination of the drug.

Similar to our case, Bihari et al. published the case of a patient with prolonged retention of awareness during CPR, stating that this rare situation might be a reflection of effective chest compressions, which were manual in their setting (10). However, Levinter et al. (11) used Thumper® (Michigan Instruments, Grand Rapids, Michigan, US) and Quinn et al. used an active compressiondecompression device in their cases of consciousness during CPR (12). In both reports, the authors concluded that the blood flow that was provided by CPR was sufficient for the adequate perfusion of the brain. Our patient was an in-hospital cardiac arrest case, and prompt CPR with Lucas $2 \AA$ was initiated when cardiac arrest with asystole was noticed. Notably, the rarity of this phenomenon in the literature and in clinical practice might raise suspicions that restoring cerebral blood flow immediately after cardiac arrest alone may not be enough to cause consciousness during CPR.

\section{Conclusion}

This is the first published E-CPR case report by emergency physicians in Turkey. Extracorporeal life support for digoxin intoxication might be a beneficial option for intoxicated patients. However, the establishment of institutional protocols and ECMO teams is essential.

\section{REFERENCES}

References

1. Ehle M, Patel C, Giugliano RP. Digoxin: Clinical highlights. A review of digoxin and its use in contemporary medicine. Crit Pathways in Cardiol, 2011, Vol. 10, pp. 9398.

2. January CT, Wann LS, Alpert JS et al. 2014 AHA/ACC/HRS Guideline for the Management of Patients With Atrial Fibrillation. Journal of American College of Cardiology, 2014, Vol. 64 (21), pp. e1-e76.

3. Gheorhgiade M, van Veldhuisen DJ, Colucci WS. Contemporary use of digoxin in the management of cardiac disorders. Circulation, 2006, Vol. 113, pp. 2556-2564. 
4. Bauman JL, Didomenico RJ, Galanter WL. Mechanisms, manifestations, and management of digoxin toxicity in the modern era. Am J Cardiovasc Drugs, 2006, Vol. 6, pp. 1-12. 5. Kagawa E. Extracorporeal cardiopulmonary resuscitation for adult cardiac arrest patients. World J Crit Care Med, 2012, Vol. 1 (2), pp. 46-49.

6. Wengenmayer $T$, Rombach $S$, Ramshorn $F$, et al. Influence of low-flow time on survival after extracorporeal cardiopulmonary resuscitation (eCPR). Crit Care. 2017, 21(1):157. doi:10.1186/s13054-017-1744-8,. Published 2017 Jun 22.

7. Farag M, Badowski D, Koschny R, Skopp G, Bricic A, Szabo GB. Extracorporeal life support and digoxin-specific Fab fragments for successful management of Taxus baccata intoxication with low output and ventricular arrhythmia. American Journal of Emergency Medicine, 2017, Vol. 35 (12), pp. 1987.e3-1987.e7.

8. Wu IL, Yu JH, Lin CC, Seak CJ, Olson KR, Chen HY. Fatal cardiac glycoside poisoning due to mistaking foxglove for comfrey. Clin Toxicol (Phila). 2017, 55(7):670-673. doi:10.1080/15563650.2017.1317350,

9. Wermuth ME, Vohra R, Bowman N, Furbee RB, Rusyniak DE. Cardiac Toxicity from Intentional Ingestion of PongPong Seeds (Cerbera Odollam). J Emerg Med. 2018, 55(4):507-511. doi:10.1016/j.jemermed.2018.05.021,.

10. Bihari S, Rajajee V. Prolonged retention of awareness during cardiopulmonary resuscitation for asystolic cardiac arrest. Neurocritical Care, 2008, Vol. 9 (3), pp. 382-6.

11. Lewinter JR, Carden DL, Nowak RM, Enriquez E, Martin GB. CPR-dependent consciousness: evidence for cardiac compression causing forward flow. Ann Emerg Med. 1989, 18(10):1111-5.

12. Quinn JV, Hebert PC, Stiell IG. Need for sedation in a patient undergoing active compression-decompression cardiopulmonary resuscitation. Acad Emerg Med. 1994, 1(5):463-6 .

Funding: None Declared

\section{Conflict of Interest: None Declared}

Corresponding Author: Yunus Emre ÖZLÜER

Adnan Menderes University Hospital Department of Emergency Medicine, Aydın/TURKEY

yeozluer@adu.edu.tr 